\title{
An Alpha-Beta Phase Diagram Representation of the Zeros and Properties of the Mittag-Leffler Function
}

\author{
John W. Hanneken, B. N. Narahari Achar, and David M. Vaught \\ Department of Physics, University of Memphis, Memphis, TN 38152, USA \\ Correspondence should be addressed to John W. Hanneken; jhannekn@memphis.edu
}

Received 8 May 2013; Accepted 25 June 2013

Academic Editor: Dumitru Baleanu

Copyright ( 2013 John W. Hanneken et al. This is an open access article distributed under the Creative Commons Attribution License, which permits unrestricted use, distribution, and reproduction in any medium, provided the original work is properly cited.

A significant advance in characterizing the nature of the zeros and organizing the Mittag-Leffler functions into phases according to their behavior is presented. Regions have been identified in the domain of $\alpha$ and $\beta$ where the Mittag-Leffler functions $E_{\alpha, \beta}(z)$ have not only the same type of zeros but also exhibit similar functional behavior, and this permits the establishment of an $\alpha$ - $\beta$ phase diagram.

\section{Introduction}

The Mittag-Leffler (ML) function $E_{\alpha, \beta}(z)$ defined by

$$
E_{\alpha, \beta}(z)=\sum_{k=0}^{\infty} \frac{z^{k}}{\Gamma(\alpha k+\beta)}, \quad z \in \mathbb{C}
$$

is a generalization of the exponential function and plays a fundamental role in the theory of fractional differential equations with numerous applications in physics. Consequently, books devoted to the subject of fractional differential equations (i.e., Podlubny [1], Magin [2], Kilbas et al. [3], and Mainardi [4]) all contain sections on the Mittag-Leffler functions. Despite the inherent importance of Mittag-Leffler functions in fractional differential equations, their behaviour and types of zeros have not been fully characterized. This work resolves this delinquency by identifying regions in the domain of $\alpha$ and $\beta$ where the Mittag-Leffler functions exhibit similar behaviour. In this work, $z$ is restricted to real numbers.

While Mittag-Leffler functions in general exhibit a diverse range of behaviors, ML functions which have the same types of zeros also exhibit many other similar properties. Hence, it is logical to organize the ML functions with similar types of zeros and similar properties into regions of the parameter space $(\alpha, \beta)$ (restricted to positive real numbers in this work) resulting in a $\alpha-\beta$ "phase diagram" for the MittagLeffler functions. Information extracted from a review of the literature on the theory of the zeros of ML functions together with the numerical results of this work yields the first depiction of the $\alpha-\beta$ phase diagram for the MittagLeffler functions shown in Figure 1 for the range $0<\alpha \leq$ 3. Seven major regions or phases have been identified and descriptively labeled by an ordered pair of symbols where the first symbol of the pair indicates the number of real zeros attributed to the ML function in that phase [i.e., none (0), finite $(f)$, or infinite $(\infty)$ ] and the second symbol of the pair refers to the number of complex zeros attributed to the Mittag-Leffler functions in that same phase.

Specifically, the seven phases are regions where the ML functions $E_{\alpha, \beta}(z)$ have (1) a finite number of real zeros and an infinite number of complex zeros $[f / \infty],(2)$ one real zero and an infinite number of complex zeros [1/o], (3) an infinite number of real zeros and no complex zeros $[\infty / 0]$, (4) an infinite number of real zeros and a finite number of complex zeros $[\infty / f]$, and (5) a special point where $E_{\alpha, \beta}(z)$ has no zeros at all $[0 / 0]$. The remaining two phases both have no real zeros and an infinite number of complex zeros $[0 / \infty]$, but exhibit different functional behaviors depending on the value of $\alpha$ and thus are denoted as (6) $[0 / \infty]_{\alpha<1}$ for the region $0<\alpha<1$ and (7) $[0 / \infty]_{\alpha>1}$ for the region $1 \leq \alpha \leq 2$. It is interesting to note that although not excluded theoretically, the phase $[\infty / \infty]$ does not seem to exist in the range considered here $0<\alpha \leq 3$. Theoretical considerations show that the phases $[0 / f],[f / 0]$, and $[f / f]$ 


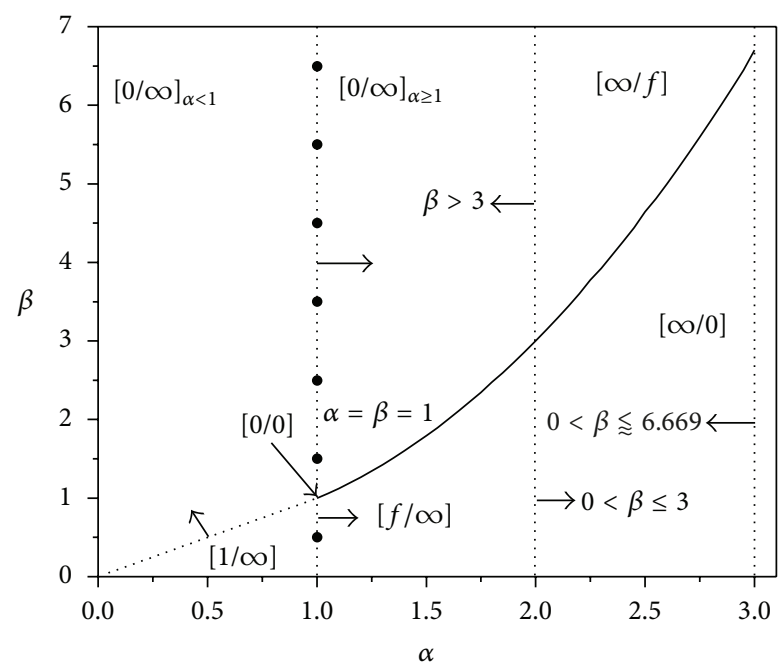

Figure 1: $\alpha-\beta$ phase diagram for $E_{\alpha, \beta}(z)$. The arrows point to the particular phase into which a Mittag-Leffler function whose parameters lie on a phase boundary belongs. Dotted lines are used to indicate phase boundaries where $\alpha$ and $\beta$ are known exactly and solid lines for boundaries where $\alpha$ and/or $\beta$ may be determined as accurately as desired but not known exactly. In opposition to the remainder of the points on the line $\alpha=1$, the dark points at $\beta=3 / 2,5 / 2,7 / 2,9 / 2, \ldots$ belong to phase $[0 / \infty]_{\alpha<1}$ and $\beta=1 / 2$ to phase $[1 / \infty]$.

are to be excluded. After some theoretical arguments which are applicable to the zeros of all Mittag-Leffler functions, each phase will be discussed separately with the corresponding supporting literature references. Examples will be provided for the typical behavior of the ML functions belonging to each of these phases.

\section{Zeros of $E_{\alpha, \beta}(z)$ Theory}

It follows from Hadamard's factorization theory that an entire function of fractional order has infinitely many zeros (Ahlfors [5]). The Mittag-Leffler function given by (1) is an entire function of order $1 / \alpha$ (Gorenflo and Mainardi [6]). Consequently, all ML functions $E_{\alpha, \beta}(z)$ will have an infinite number of zeros with the possible exception of when $1 / \alpha$ is an integer (i.e., when $\alpha=1,1 / 2,1 / 3,1 / 4, \ldots$ ). Note that Hadamard's statement says nothing about the number of zeros when $1 / \alpha$ is an integer. In these cases, there may be no zeros, a finite number of zeros, or an infinite number of zeros. However, Sedletski [7, 8] has shown that with the exception of $\alpha=\beta=1$ the Mittag-Leffler functions have an infinite number of zeros for $\beta>0$. Since all terms in (1) are positive for positive $z$, the Mittag-Leffler functions have no real zeros for $z>0$. In addition, all complex zeros occur as pairs of complex conjugates (Gorenflo et al. [9]). A systematic description of the various phases follows.

\section{Phase $[0 / 0]$}

The ML function $E_{1,1}(z)$ is equivalent to the exponential function $e^{z}$ and is the only Mittag-Leffler function which has no zeros (Sedletski [7]) and thus is just a single point in the phase diagram. The fact that $E_{1,1}(z)$ does not have an infinite number of zeros is consistent with Hadamard's statement since $1 / \alpha$ is an integer.

\section{Phase $[1 / \infty]$}

This phase extends from $0<\alpha<1$ and $0<\beta<\alpha$ with different behaviors on the boundaries as follows: the ML functions whose parameters lie on the vertical dotted line at $\alpha=1$ which separates the $[1 / \infty]$ phase from $[f / \infty]$ belong to the $[f / \infty]$ phase (with the single exception of $E_{1,1 / 2}(z)$ which belongs to $[1 / \infty]$ - see Section 8 for details), while the ML functions whose parameters lie on the dotted line $\alpha=\beta$ belong to the $[0 / \infty]$ phase. Figure 2 shows a typical MittagLeffler function in this phase.

The function is positive for positive $z$, and as $z \rightarrow$ $-\infty$, the function crosses the negative $z$-axis only once and asymptotically approaches zero from below. This is evident from the asymptotic expansion of $E_{\alpha, \beta}(z)$ given by Podlubny [1]:

$$
E_{\alpha, \beta}(z) \approx \frac{-1}{[z \Gamma(\beta-\alpha)]}
$$

In this phase $0<\alpha<1$ and $0<\beta<\alpha$, thus $-1<\beta-\alpha<0$ and $\Gamma(\beta-\alpha)$ is negative and as $z \rightarrow-\infty, E_{\alpha, \beta}(z)$ is negative and thus approaches zero from below the negative $z$-axis. Theory confirms the fact that each $E_{\alpha, \beta}(z)$ in this phase has only one real negative simple zero (Popov and Sedletski [10]). The fact that each ML function (except for $E_{1,1}(z)$ ) has an infinite number of zeros for $\beta>0$ requires that each ML function in this phase has an infinite number of complex zeros since each function has only one real zero.

\section{Phase $[0 / \infty]_{\alpha<1}$}

This phase extends for $\beta \geq \alpha$, from $0<\alpha<1$ with the line $\alpha=\beta$ separating the phase $[1 / \infty]$ from $[0 / \infty]_{\alpha<1}$, and Mittag-Leffler functions $E_{\alpha, \alpha}(z)$ on the line belong to the phase $[0 / \infty]_{\alpha<1}$. The reason ML functions on the vertical line $\alpha=1$ are not in this phase will be discussed in detail in the next section. Figure 3 shows the graph of a typical MittagLeffler function in the phase $[0 / \infty]_{\alpha<1}$.

The function is positive for positive $z$ and remains positive while asymptotically approaching the negative real axis as $z \rightarrow-\infty$ and thus has no real zeros. This is evident by applying (2) with $\beta>\alpha, \Gamma(\beta-\alpha)$ is then positive and as $z \rightarrow-\infty E_{\alpha, \beta}(z)$ is positive and thus approaches zero from above the negative real axis. For $\alpha=\beta$, the asymptotic expansion of $E_{\alpha, \alpha}(z)$ as $z \rightarrow-\infty$ is given by Podlubny [1]:

$$
E_{\alpha, \alpha}(z) \approx-\frac{1}{\left[z^{2} \Gamma(-\alpha)\right]} .
$$

For $0<\alpha<1, \Gamma(-\alpha)$ is negative, and as $z \rightarrow-\infty E_{\alpha, \alpha}(z)$ is positive and this function also approaches zero from above the negative real axis. The behaviour of the Mittag-Leffler function in this phase is confirmed by the theoretical work of 


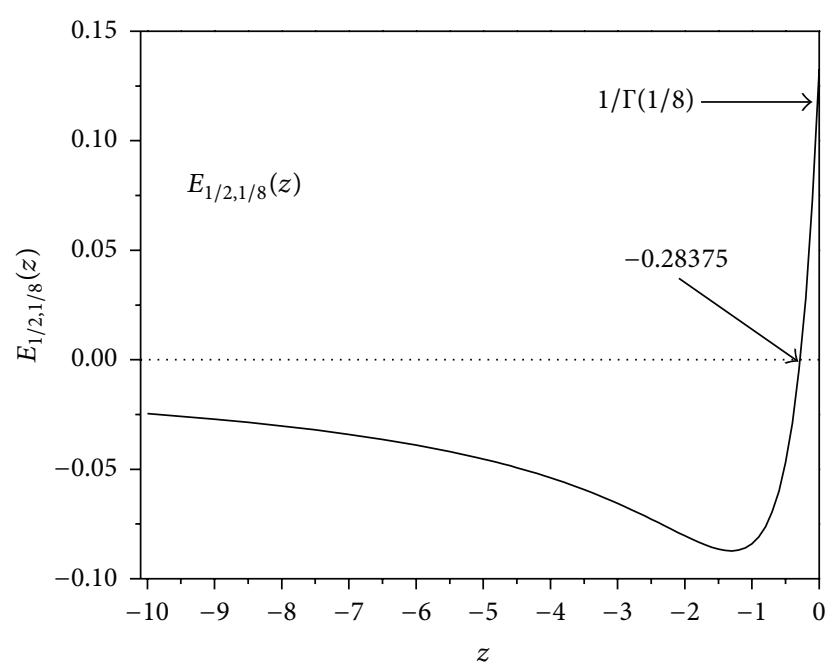

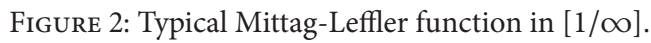

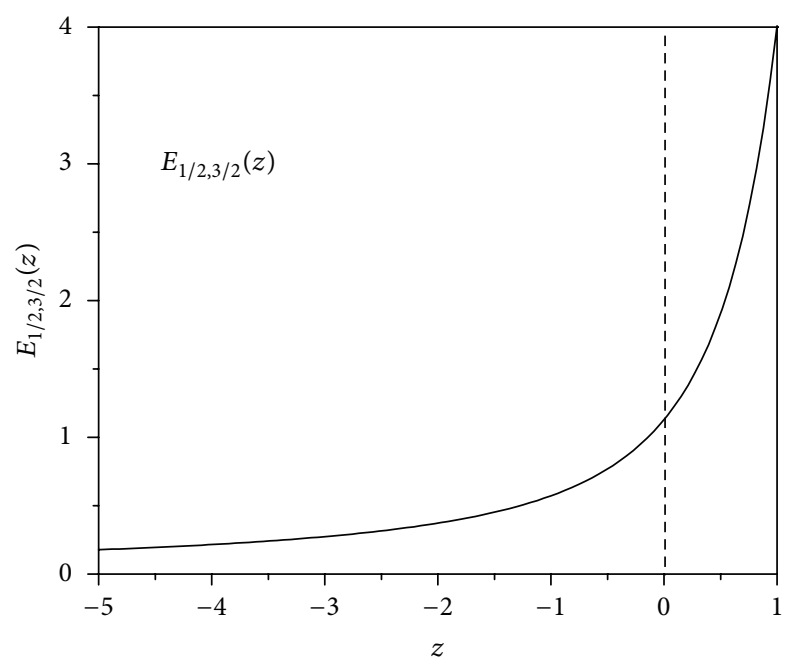

Figure 3: Typical Mittag-Leffler function in $[0 / \infty]_{\alpha<1}$.

Schneider [11] and Miller and Samko [12, 13] who proved that for $0<\alpha \leq 1$ and $\beta \geq \alpha, E_{\alpha, \beta}(z)$ is a complete monotonic nonnegative function on the negative $z$-axis. Furthermore, Aleroev and Aleroeva [14] have proven that all zeros of $E_{\alpha, \alpha}(z)$ are complex for $0<\alpha<1$. Since the Mittag-Leffler functions in this phase have no real zeros, then they must have an infinite number of complex zeros.

\section{Phase $[0 / \infty]_{\alpha \geq 1}$}

This phase extends from $1 \leq \alpha \leq 2$ to the phase boundary separating phase $[0 / \infty]_{\alpha \geq 1}$ from $[f / \infty]$. This latter phase boundary does not appear to be a simple function and will be examined in detail in the discussion of phase $[f / \infty]$. All Mittag-Leffler functions in phase $[0 / \infty]_{\alpha \geq 1}$ are characterized by $\beta>\alpha$. Figure 4 shows a typical Mittag-Leffler function in this phase.

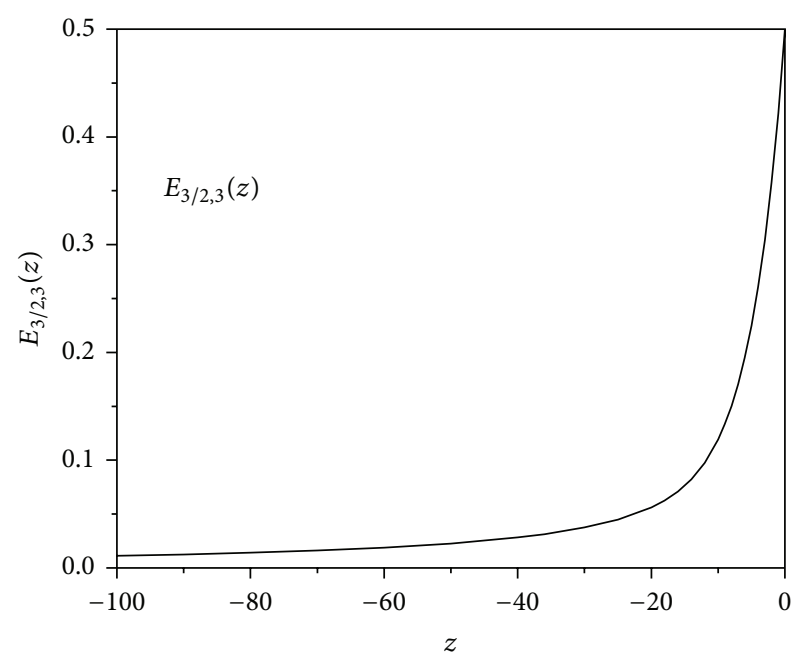

FIGURE 4: Typical Mittag-Leffler function in phase $[0 / \infty]_{\alpha \geq 1}$.

As with phase $[0 / \infty]_{\alpha<1}$, the Mittag-Leffler function is positive for positive $z$ and remains positive while asymptotically approaching the negative real axis as $z \rightarrow-\infty$ and thus has no real zeros. However, the Mittag-Leffler function in phase $[0 / \infty]_{\alpha \geq 1}$ exhibits a fundamentally different functional behaviour from the Mittag-Leffler functions in phase $[0 / \infty]_{\alpha<1}$. This can be understood by recognizing that Mittag-Leffler functions for which $\alpha+1>\beta$ can be decomposed into two parts [15] given by

$$
E_{\alpha, \beta}(-z)=g_{\alpha, \beta}(-z)+f_{\alpha, \beta}(-z),
$$

where

$$
\begin{aligned}
& g_{\alpha, \beta}(-z)=\left(\frac{2}{\alpha} \exp \left[z^{1 / \alpha} \cos \left(\frac{\pi}{\alpha}\right)\right]\right. \\
& \left.\quad \times \cos \left[\frac{\pi(1-\beta)}{\alpha}+z^{1 / \alpha} \sin \left(\frac{\pi}{\alpha}\right)\right]\right) \\
& \quad \times\left(z^{(\beta-1) / \alpha}\right)^{-1}, \\
& f_{\alpha, \beta}(-z) \quad\left(\frac { 1 } { \pi } \int _ { 0 } ^ { \infty } \left\{\left(\exp \left(-z^{1 / \alpha}\right) r^{\alpha-\beta}\left[r^{\alpha} \sin (\pi \beta)+\sin [\pi(\beta-\alpha)]\right]\right)\right.\right. \\
& \left.\left.\quad \times\left(r^{2 \alpha}+2 r^{\alpha} \cos (\pi \alpha)+1\right)^{-1}\right\} d r\right) \\
& \quad \times\left(z^{(\beta-1) / \alpha}\right)^{-1} .
\end{aligned}
$$

Thus, Mittag-Leffler functions for which $\alpha+1>\beta$ are composed of a function $f_{\alpha, \beta}(-z)$ which asymptotically approaches the negative $z$ axis as $-z \rightarrow \infty$ superimposed on an oscillatory function $g_{\alpha, \beta}(-z)$ [15]. For Mittag-Leffler 


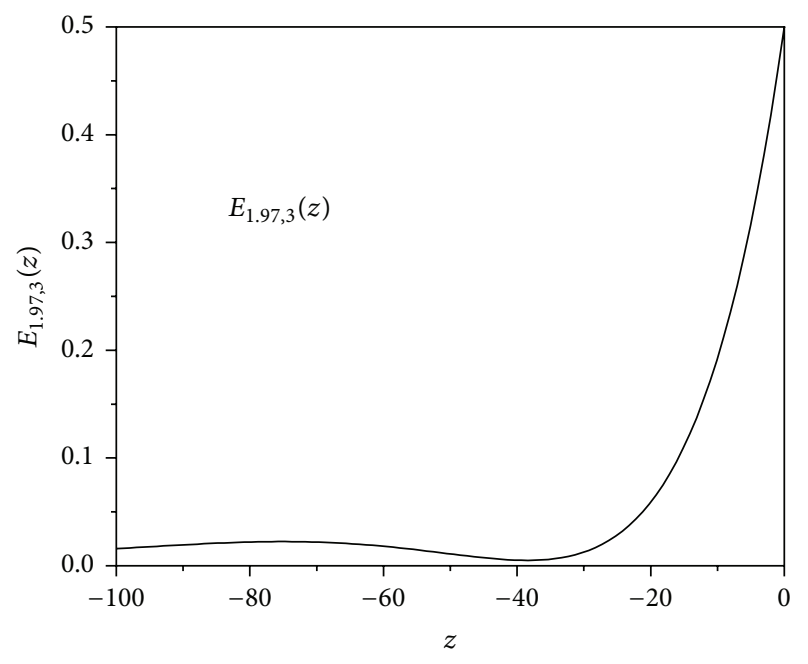

Figure 5: An ML function from phase $[0 / \infty]_{\alpha>1}$ displaying the oscillatory behaviour as $z \rightarrow-z$.

functions in which $\alpha+1 \geq \beta$, there exists the following recursion relation:

$$
E_{\alpha, \beta}(z)=z^{-m} E_{\alpha, \beta-\alpha m}(z)-\sum_{k=1}^{m} \frac{z^{-k}}{\Gamma(\beta-\alpha k)}, \quad m=1,2,3, \ldots
$$

This permits representing any Mittag-Leffler function with $\alpha+1 \geq \beta$ in terms of a Mittag-Leffler function with $\alpha+1>\beta$, which in turn can then be represented by (5). For example, using the recursion relation with $m=2, E_{3 / 2,4}(z)$ can be calculated from $E_{3 / 2,1}(z)$ as follows:

$$
E_{3 / 2,4}(z)=z^{-2} E_{3 / 2,1}(z)-\frac{1}{z \Gamma(5 / 2)}-\frac{1}{z^{2}}
$$

As with the previous example, any Mittag-Leffler function in phase $[0 / \infty]_{\alpha \geq 1}$ can be represented in terms of a MitttagLeffler function which can be calculated using (5). Consequently, although not always apparent, inherent in all Mittag-Leffler functions in phase $[0 / \infty]_{\alpha \geq 1}$ is the oscillatory behaviour of $g_{\alpha, \beta}(-z)$ in (5). Figure 5 shows a MittagLeffler function in phase $[0 / \infty]_{\alpha \geq 1}$ in which this oscillatory behaviour is clearly evident.

The fact that $g_{\alpha, \beta}(-z)$ is zero for $0<\alpha<1$ [15] is why the Mittag-Leffler functions of phase $[0 / \infty]_{\alpha<1}$ exhibit no oscillatory behaviour and why phases $[0 / \infty]_{\alpha<1}$ and $[0 / \infty]_{\alpha \geq 1}$ are fundamentally different phases. Although $g_{\alpha, \beta}(-z)$ is zero for $0<\alpha<1$, it is instructive to ask if $g_{\alpha, \beta}(-z)$ is ever equal to zero when $\alpha \geq 1$. Since $z$ is in the cosine argument, for a fixed $\alpha$ and $\beta, g_{\alpha, \beta}(-z)$ can only be zero if $\sin (\pi / \alpha)$ is zero. This limits the possibilities to the vertical line $\alpha=1$ whereby $g_{\alpha, \beta}(-z)=2 e^{-z} \cos [\pi(1-\beta)]$ which is zero when $\beta=1 / 2,3 / 2,5 / 2, \ldots$. Thus, at these isolated points $\alpha=1, \beta=1 / 2,3 / 2,5 / 2, \ldots$ the oscillatory nature of $g_{\alpha, \beta}(-z)$ is absent. Consequently, for $\beta>1$, these points belong to phase $[0 / \infty]_{\alpha<1}$ and all other points on the vertical line belong to phase $[0 / \infty]_{\alpha \geq 1}$ as indicated by the arrow in Figure 1. Similarly, for $\beta<1$, the point $\beta=$ $1 / 2$ belongs to phase $[1 / \infty]$. To highlight this distinction, these special points are shown as dark points in Figure 1. The fact that, according to Schneider [11] and Miller and Samko $[12,13]$, ML functions on the line $\alpha=1$ with $\beta>1$ are complete monotonic nonnegative functions on the negative $z$-axis simply means that in this case the $f_{\alpha, \beta}(-z)$ function in (5) dominates and does not permit the oscillatory nature of $g_{\alpha, \beta}(-z)$ to be manifested.

For $1 \leq \alpha \leq 2$, the phase $[0 / \infty]_{\alpha \geq 1}$ includes all MittagLeffler functions above the phase boundary line but not those on the line. This last fact will be discussed in detail in the following section. Mittag-Leffler functions $E_{2 \cdot \beta}(z)$ on the vertical boundary line $\alpha=2$ separating phase $[0 / \infty]_{\alpha \geq 1}$ from $[\infty / f]$ for $\beta>3$ belong to phase $[0 / \infty]_{\alpha \geq 1}$ as indicated by the arrow in Figure 1. This is confirmed by the theoretical work of Popov and Sedletski [10] who show that $E_{2, \beta}(z)$ has no real zeros for $\beta>3$. With no real zeros, each ML function in this phase must have an infinite number of complex zeros.

\section{Phase $[f / \infty]$}

This phase extends from $1 \leq \alpha<2$ and from $\beta>0$ to the $[0 / \infty]_{\alpha \geq 1}$ phase boundary line in Figure 1. Figure 6 shows the graph of a typical Mittag-Leffler function belonging to this phase.

The function is positive for positive $z$ and as $z \rightarrow-\infty$, the function oscillates crossing the negative $z$-axis a finite number of times before asymptotically approaching zero. This general behavior applies to all Mittag-Leffler functions in this phase. However, the Mittag-Leffler functions within this phase differ with respect to how they asymptotically approach zero. Some approach from above the negative $z$ axis while others approach from below. Those Mittag-Leffler functions that approach from below must have crossed the $z$-axis an odd number of times. For example, $E_{\alpha, 1}(z)$ approaches from below for $1<\alpha<2$ and thus has an odd number of negative real zeros (Wiman [16], Gorenflo and Mainardi [15]). This is evident from (2) since $\beta=1$ and $\Gamma(1-\alpha)$ is negative for $1<\alpha<2$, therefore $E_{\alpha, 1}(z)$ is negative as $z \rightarrow-\infty$. Similarly, $E_{\alpha, \alpha}(z)$ for $1<\alpha<2$ approaches zero from below and has on odd number of negative real zeros. This is evident from (3) since $\Gamma(-\alpha)$ is positive for $1<\alpha<2$ and thus $E_{\alpha, \alpha}(z)$ is negative as $z \rightarrow-\infty$. Those Mittag-Leffler functions that approach zero from above the negative real axis must have crossed the $z$-axis an even number of times. For example, $E_{\alpha, 2}(z)$ for $1<\alpha<2$ approaches zero from above. This is evident from (2) since $\beta=2$ and $\Gamma(2-\alpha)$ is positive for $1<\alpha<2$, therefore $E_{\alpha, 2}(z)$ is positive as $z \rightarrow-\infty$ and has an even number of negative real zeros. With only a finite number of real zeros, each ML function in this phase must have an infinite number of complex zeros.

Consequently, the phase $[f / \infty]$ can be subdivided into regions where the Mittag-Leffler function has either an even or an odd number of real zeros. There are two regions where the Mittag-Leffler function has an even number of real zeros, and these two regions will be referred to as $[f($ even $) / \infty]$. There is one region where the Mittag-Leffler 


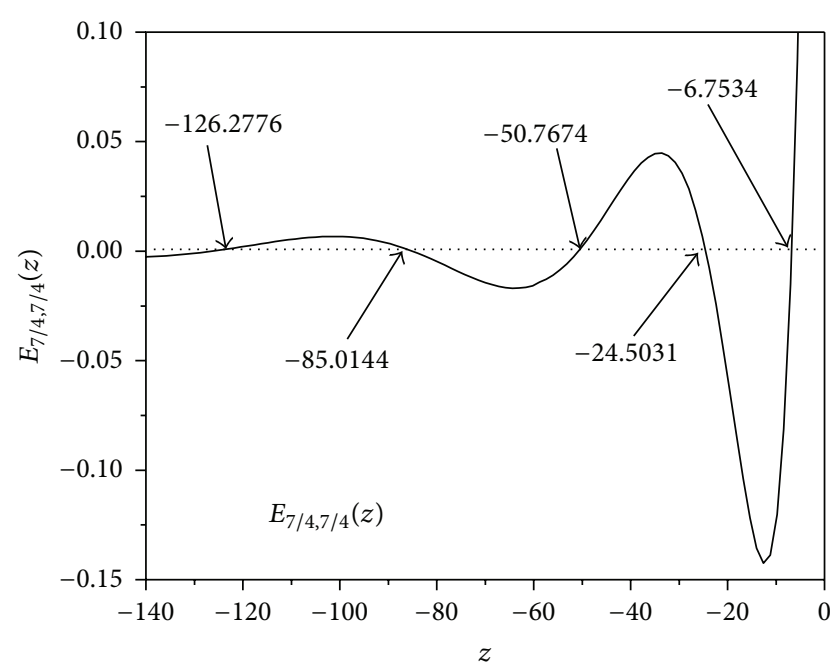

Figure 6: Typical Mittag-Leffler function in [ $f / \infty]$.

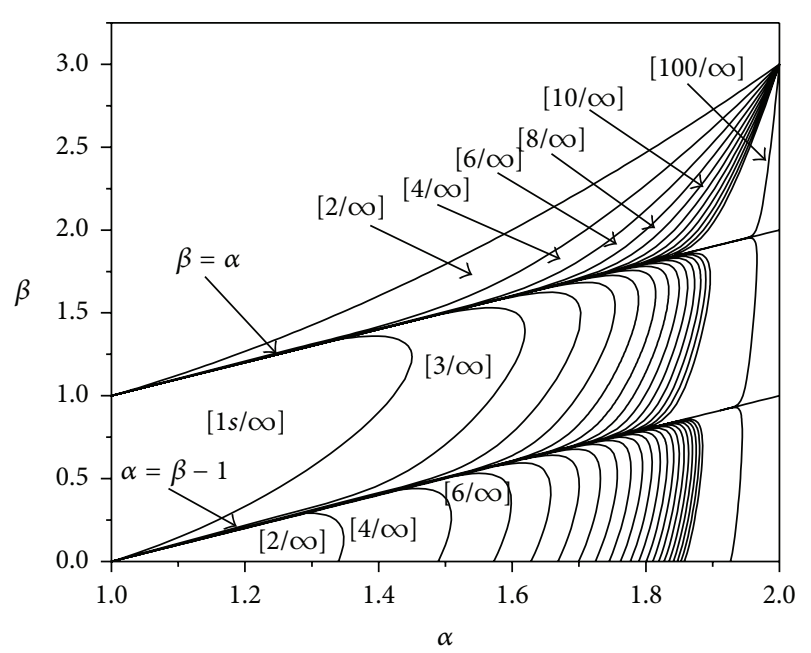

Figure 7: Phase $[f / \infty]$ showing the two regions $[f($ even $) / \infty]$ with subphases $[n / \infty], n=2,4,6,8, \ldots$ and the one region $[f($ odd $) / \infty]$ with subphases $[n / \infty], n=1,3,5,7, \ldots$.

function has an odd number of real zeros, and it will be referred to as $[f($ odd $) / \infty]$. Phase $[f / \infty]$ has been extensively mapped by the authors and is shown in Figure 7 . The regions $[f($ even $) / \infty]$ and $[f$ (odd)/ $/ \infty]$ will be discussed in more detail in the next two sections.

\section{Region $[f($ odd $) / \infty]$}

This region, which extends from $1 \leq \alpha<2$, is bounded below by the line $\beta=\alpha-1$ and is bounded above by the line $\beta=\alpha$ as shown in Figure 8. Depicted in Figure 8 are the lines separating the various subphases $[n / \infty]$ where the MittagLeffler function has $n=1,3,5,7, \ldots$ zeros. Lines begin on the line $\beta=\alpha$ curl around and end at the point $\alpha=\beta=1$. Each subsequent line is asymptotically closer to the $\beta=\alpha-1$ line as it approaches the point $\alpha=\beta=1$. The number of subphases drawn in Figure 8 was determined by when there was no clear

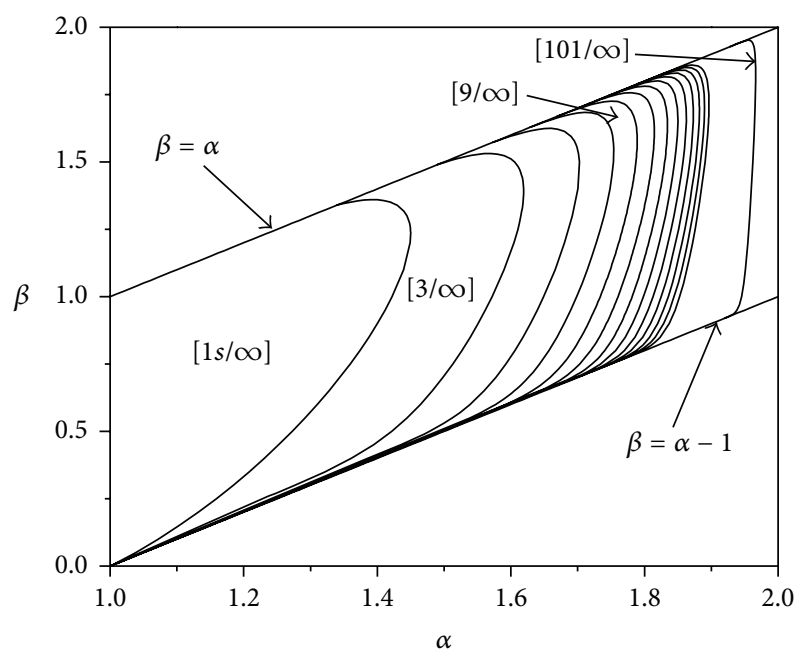

FIGURE 8: Region $[f$ (odd)/ $\infty$ ] showing the subphases $[n / \infty], n=$ $1 s, 3,5,7,9, \ldots$.

separation distinguishing consecutive subphases. The line separating subphase $[101 / \infty]$ from $[103 / \infty]$ is also drawn for perspective.

Points on the boundary lines separating consecutive subphases can be determined numerically as accurately as desired, but no exact values are known (other than the point $\alpha=\beta=1$ ). Two points on each curve will be given as reference values which are accurate to 11 significant digits. Points on the line $\alpha=\beta$ where the boundary line separates subphase $[n / \infty]$ from subphase $[(n+2) / \infty]$ for $n=1,3,5, \ldots 101$ are given in Table 3(a). Additional reference values are given in Table 3(b) which lists points on the horizontal line $\beta=$ 1 where the boundary line separates subphase $[n / \infty]$ from subphase $[(n+2) / \infty]$ for $n=1,3,5, \ldots 101$. These reference values have been investigated by Hanneken et al. [17] who published a partial table including values of $\alpha$ up to $n=$ 11281. The values of $\alpha$ are recorded such that the MittagLeffler function with that value of $\alpha$ and $\beta$ will be in subphase $[n / \infty]$ whereas by adding one digit to the least significant digit of $\alpha$ the corresponding Mittag-Leffler function will then be in subphase $[(n+2) / \infty]$.

It should be noted that subphase $[1 / \infty]$ within region [ $f$ (odd) $/ \infty]$ must be distinguished from phase $[1 / \infty]$ to which it is adjacent on the phase diagram. The graphs of Mittag-Leffler functions in both subphase [1s/o] and phase $[1 / \infty]$ cross the $z$-axis only once and asymptotically approach the negative $z$-axis from below as $z \rightarrow-\infty$.

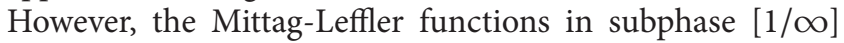
exhibit a fundamentally different functional behaviour from the Mittag-Leffler functions in phase $[1 / \infty]$. Since $\alpha+$ $1>\beta$ for all Mittag-Leffler functions in both phase [1/ $\infty]$ and subphase $[1 / \infty]$, the decomposition into the functions $g_{\alpha, \beta}(-z)$ and $f_{\alpha, \beta}(-z)$ in (5) is valid. However, in phase

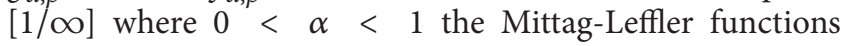
are entirely described by the function $f_{\alpha, \beta}(-z)$, and the oscillatory function $g_{\alpha, \beta}(-z)$ is absent. This fundamental difference requires subphase $[1 / \infty]$ be distinguished from 
phase $[1 / \infty]$. Consequently, subphase $[1 / \infty]$ will be denoted as $[1 s / \infty]$ ( $s$ for subphase) as indicated in Figure 8 . This is the only subphase which requires a special notation. All the points on the line $\alpha=1$ for $0<\beta<1$ belong to subphase [1s/o] with one exception. The point $\alpha=1, \beta=1 / 2$ belongs to phase $[1 / \infty]$ and not to subphase $[1 s / \infty]$ as discussed in detail in Section 6.

\section{Region $[f($ even $) / \infty]$}

Region $[f$ (even)/ $\infty$ ] appears both above and below region $[f$ (odd) $/ \infty]$ on the phase diagram as shown in Figure 7. Although separated in phase space, the two regions of $[f($ even $) / \infty]$ are fundamentally the same in that the MittagLeffler functions in both regions have similar behaviour. The lower region, which extends from $1<\alpha<2$, is bounded below by the line $\beta=0$ and is bounded above by the line $\beta=\alpha-1$ as shown in Figure 9. Depicted in Figure 9 are the lines separating the various subphases $[n / \infty]$ where the Mittag-Leffler function has $n=2,4,6,8, \ldots$ zeros. Lines begin on the line $\beta=\alpha-1$ curl around and end on the line $\beta=0$. The number of subphases drawn in Figure 7 was determined by when there was no clear separation distinguishing consecutive subphases. The line separating subphase $[100 / \infty]$ from $[102 / \infty]$ is also drawn for perspective. Points on the boundary lines separating consecutive subphases can be determined numerically as accurately as desired, but no exact values are known. Two points on each curve will be given as reference values which are accurate to 11 significant digits. Points on the line $\beta=$ $\alpha-1$ where the boundary line separates subphase $[n / \infty]$ from subphase $[(n+2) / \infty]$ for $n=2,4,6, \ldots 102$ are given in Table 3(c). Additional reference values are given in Table 3(d) which lists points on the horizontal line $\beta=0$ where the boundary line separates subphase $[n / \infty]$ from subphase $[(n+$ $2) / \infty]$ for $n=2,4,6, \ldots 102$. Note that the tabulated values in Tables 3(a) and 3(d) are related. This is because $E_{\alpha, 0}(z)=$ $z E_{\alpha, \alpha}(z)$ and consequently all zeros of $E_{\alpha, \alpha}(z)$ are also zeros of $E_{\alpha, 0}(z)$ except that $E_{\alpha, 0}(z)$ has an additional zero at $z=0$. Thus, whereas $E_{\alpha, \alpha}(z)$ has an odd number of zeros, the added zero gives $E_{\alpha, 0}(z)$ an even number of zeros.

The other portion of the phase diagram in the phase $[f($ even $) / \infty]$ extends from $1<\alpha<2$ and is bounded below by the line $\alpha=\beta$ and bounded above by the $[f / \infty]$ to $[0 / \infty]$ phase boundary line as shown in Figure 10. Depicted in Figure 10 are the lines separating the various subphases $[n / \infty]$ where the Mittag-Leffler function has $n=2,4,6, \ldots$ zeros. The boundary lines begin at the point $\alpha=2, \beta=3$ and end at the point $\alpha=\beta=1$. Each subsequent line is asymptotically closer to the $\alpha=\beta$ line as it approaches the point $\alpha=\beta=1$. The number of subphases drawn in Figure 8 was determined by when there was no clear separation distinguishing consecutive subphases. The line

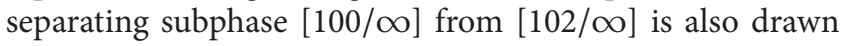
for perspective. Points on the boundary lines separating the consecutive subphases can be determined numerically as accurately as desired, but no exact values are known other than the two end points. An additional point on each curve

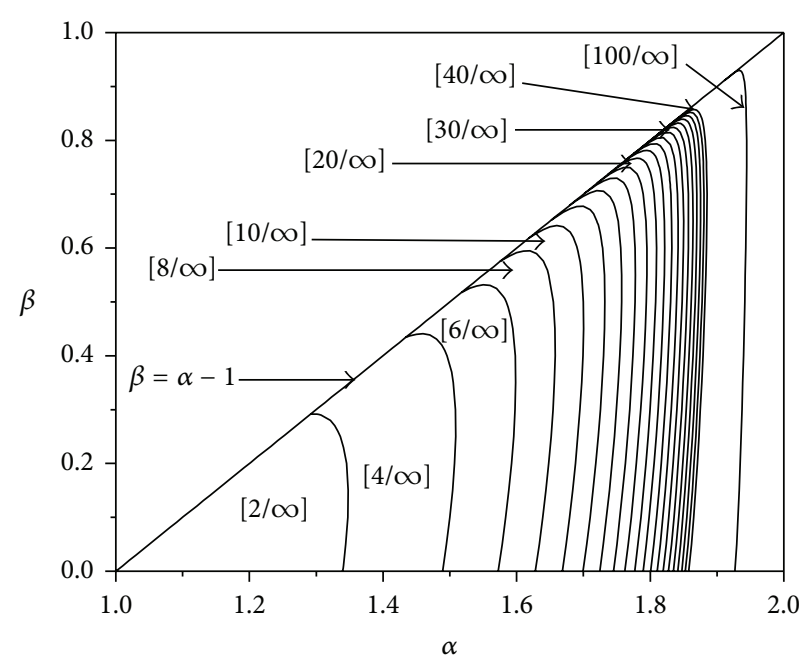

Figure 9: The lower region of $[f($ even $) / \infty]$ showing subphases $[n / \infty], n=2,4,6,8, \ldots$

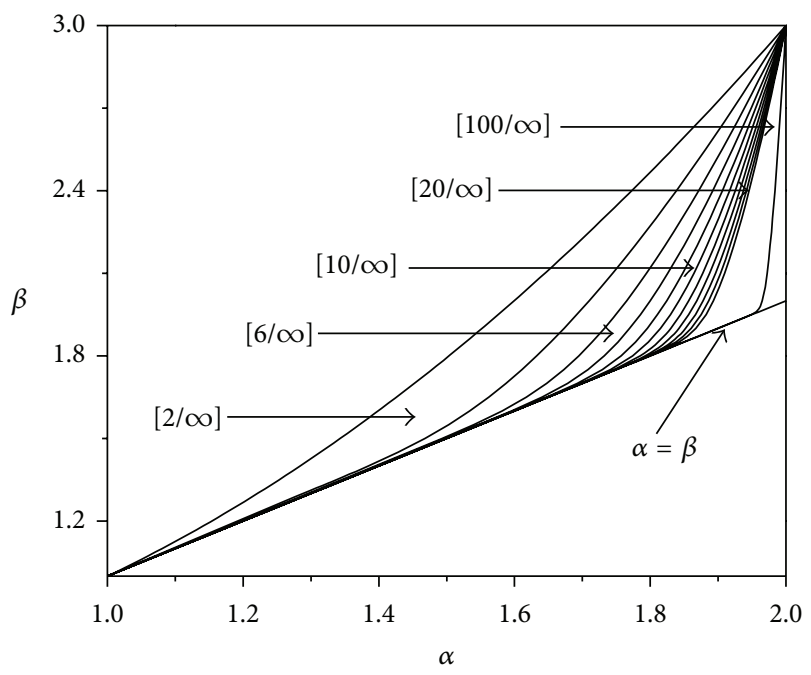

FIGURE 10: The upper region of $[f($ even $) / \infty]$ showing subphases $[n / \infty], n=2,3,4,6, \ldots$

will be given as reference values which are accurate to 11 significant digits. Points on the line $\beta=2$ where the boundary line separates subphase $[n / \infty]$ from subphase $[n+2 / \infty]$ for $n=2,4,6,8, \ldots 102$ are given in Table 3(e).

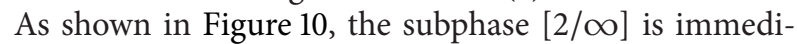
ately adjacent to the boundary line separating the $[f / \infty]$ from the $[0 / \infty]_{\alpha>1}$ phase. The Mittag-Leffler function in

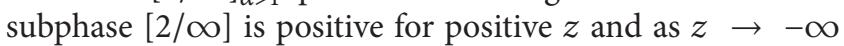
the function crosses the negative $z$-axis reaching a minimum value below the $z$-axis and then crosses the negative $z$ axis a second time and asymptotically approaches zero from above the negative $z$-axis as $z \rightarrow-\infty$. For a fixed $\alpha$, increasing $\beta$ raises the minimum value upward toward the $z$ axis causing the separation between the two zeros to decrease until the minimum value is on the $z$-axis and the two zeros have coalesced into one real zero with a double multiplicity. 


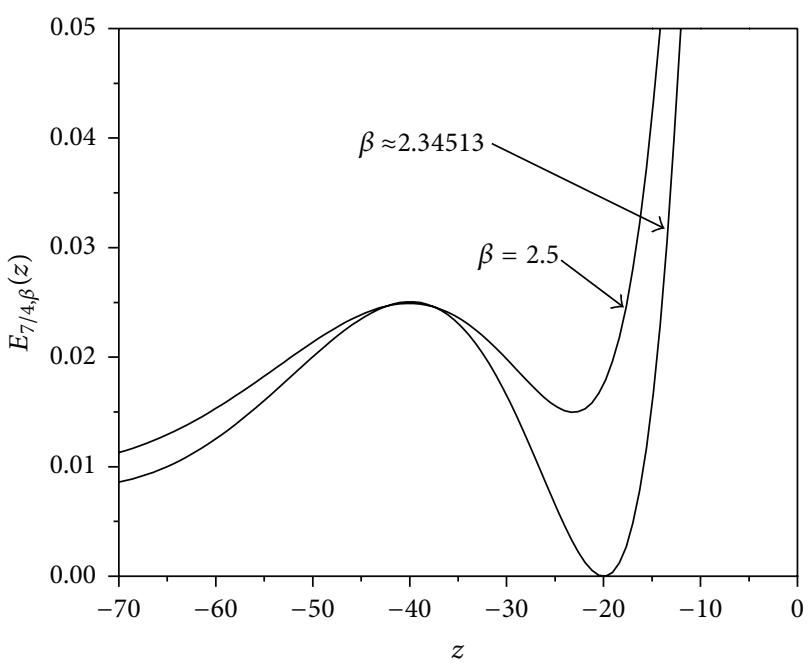

FIGURE 11: $E_{7 / 4, \beta}(z)$ for $\beta=2.34513$ and $\beta=2.5$.

This is illustrated in Figure 11 with the Mittag-Leffler function $E_{\alpha, \beta}(z)$ with $\alpha=7 / 4$. For $\beta$ just less than 2.34513 , the function has two zeros centered around $z=-20$ and increasing $\beta$ to $\approx 2.34513$ results in one real zero with a double multiplicity. A further increase in $\beta$ yields a function with no real zeros which oscillates as it asymptotically approaches $z=-\infty$ which is characteristic of a function in phase $[0 / \infty]_{\alpha \geq 1}$ as discussed earlier.

The value of $\beta$ above which the function has no real zeros delineates the boundary between the phases $[f / \infty]$ and $[0 / \infty]$ for $1<\alpha<2$, and Table 1 lists these approximate values for various values of $\alpha$ (see the discussion of phase $[\infty / f]$ for the methodology in the determination of the $\beta$ values for $2<\alpha \leq 3$ ).

A cubic equation was found to be an approximate fit to the boundary line for $1 \leq \alpha \leq 3$.

$$
\beta \approx 0.63287-0.43869 \alpha+0.79965 \alpha^{2}+0.00574 \alpha^{3} .
$$

The maximum deviation of the value of $\beta$ from Table 1 compared to that calculated from (8) is \pm 0.00153 at $\alpha=$ 1.10 ; however, for $\alpha \geq 1.85$ the maximum deviation is \pm 0.0002 . Although the points $(\alpha, \beta)$ on the phase boundary have been given to 6 significant digits in Table 1, they can be determined to much greater accuracy. In particular, for the special cases whenever $\alpha$ and/or $\beta$ are integer, these more accurately determined values can be used as reference points on the boundary and are given in Table 2 .

Although the curve extends to higher $\alpha$, it is limited to $\alpha=$ 3 in this investigation. With the exception of $\alpha=1$ and $\alpha=2$, the points which constitute the boundary line are indeterminate. For example, for $\alpha=1.5991153, E_{\alpha, 2}(z)$ has two real zeros and an infinite number of complex zeros and belongs to the phase $[f / \infty]$. Whereas for $\alpha=1.5991152$, $E_{\alpha, 2}(z)$ has no real zeros and an infinite number of complex zeros and belongs to the phase $[0 / \infty]$. Consequently, the point on the boundary line at $\beta=2$ which separates the phases $[f / \infty]$ and $[0 / \infty]$ is in the range $1.5991152<$ $\alpha<1.5991153$. Although this range can be made narrower
TABLE 1: Boundary line data points.

\begin{tabular}{|c|c|}
\hline$\alpha$ & $\beta$ \\
\hline 1.00 & 1.00000 \\
\hline 1.05 & 1.05924 \\
\hline 1.10 & 1.12400 \\
\hline 1.15 & 1.19325 \\
\hline 1.20 & 1.26674 \\
\hline 1.25 & 1.34437 \\
\hline 1.30 & 1.42608 \\
\hline 1.35 & 1.51187 \\
\hline 1.40 & 1.60173 \\
\hline 1.45 & 1.69565 \\
\hline 1.50 & 1.79365 \\
\hline 1.55 & 1.89573 \\
\hline 1.60 & 2.00191 \\
\hline 1.65 & 2.11219 \\
\hline 1.70 & 2.22660 \\
\hline 1.75 & 2.34513 \\
\hline 1.80 & 2.46779 \\
\hline 1.85 & 2.59460 \\
\hline 1.90 & 2.72557 \\
\hline 1.95 & 2.86070 \\
\hline 2.00 & 3.00000 \\
\hline 2.05 & 3.14347 \\
\hline 2.10 & 3.29112 \\
\hline 2.15 & 3.44297 \\
\hline 2.20 & 3.59900 \\
\hline 2.25 & 3.75923 \\
\hline 2.30 & 3.92367 \\
\hline 2.35 & 4.09231 \\
\hline 2.40 & 4.26516 \\
\hline 2.45 & 4.44222 \\
\hline 2.50 & 4.62350 \\
\hline 2.55 & 4.80899 \\
\hline 2.60 & 4.99871 \\
\hline 2.65 & 5.19265 \\
\hline 2.70 & 5.39082 \\
\hline 2.75 & 5.59321 \\
\hline 2.80 & 5.79984 \\
\hline 2.85 & 6.01069 \\
\hline 2.90 & 6.22578 \\
\hline 2.95 & 6.44511 \\
\hline 3.00 & 6.66867 \\
\hline
\end{tabular}

(see Table 2), the exact point that separates the phases is most likely indeterminate. Consequently, it is meaningless to discuss Mittag-Leffler functions on this phase boundary (with the exception of the two points known exactly: $\alpha=\beta=$ 1 and $\alpha=2, \beta=3$ ). It must be noted that all approximate values of $\beta$ in Tables 1 and 2 correspond to Mittag-Leffler functions in phase $[f / \infty]$ for $1<\alpha<2$ and in phase $[\infty / 0]$ for $2 \leq \alpha \leq 3$ and by adding one digit to the least significant digit of $\beta$ the function appears on the opposite side of 
TABLE 2: Boundary line reference points.

\begin{tabular}{lc}
\hline$\alpha$ & $\beta$ \\
\hline 1 & 1 \\
1.59911520632302 & 2 \\
2 & 3 \\
2.32278477412617 & 4 \\
2.60033449274294 & 5 \\
2.84748677935047 & 6 \\
3 & 6.6686719145721 \\
\hline
\end{tabular}

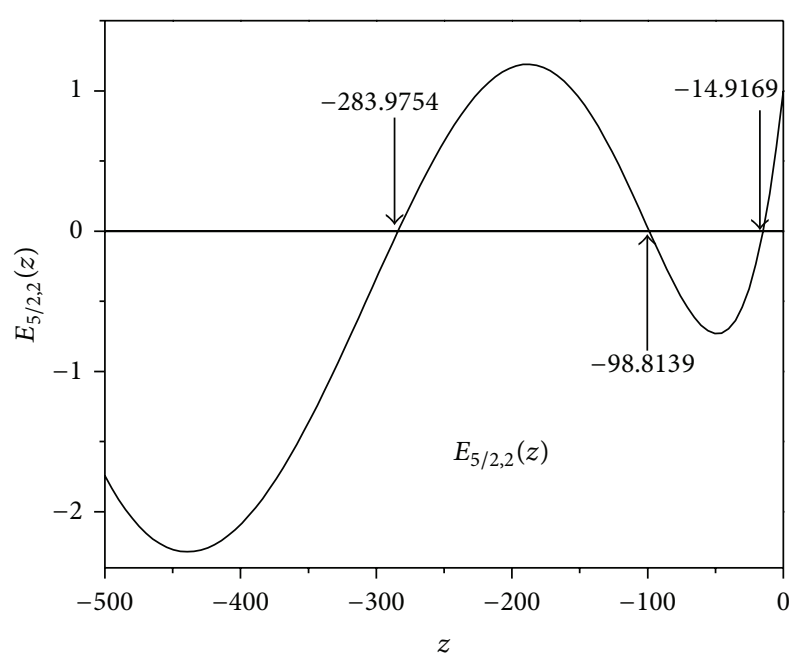

FIgURe 12: Typical Mittag-Leffler function in [ $\infty / 0]$.

the boundary line in phase $[0 / \infty]_{\alpha \geq 1}$ for $1<\alpha<2$ or phase $[\infty / f]$ for $2 \leq \alpha \leq 3$. While the opposite is true for the approximate values of $\alpha$ in Table 2 which are in either phase $[0 / \infty]_{\alpha \geq 1}$ or $[\infty / f]$ initially and after adding one digit to the least significant digit of $\alpha$ are in phase $[f / \infty]$ or $[\infty / 0]$.

It should be noted that this phase boundary line was numerically determined first by Hanneken et al. [18] in the first attempt at an alpha-beta phase diagram. This present work is more complete including a phase not originally present and includes all the subphases in $[f / \infty]$. The recent work of Duan et al. [19] shows this phase boundary line accurately for $0 \leq \alpha \leq 1$ and approximately for $\alpha>1$ but divides the entire $\alpha$ - $\beta$ phase space into only two phases and the description of his F phase having no real zeros is incorrect.

\section{Phase $[\infty / 0]$}

This phase extends from $2 \leq \alpha \leq 3$ and from $\beta>0$ to the boundary line shown in Figure 1. Figure 12 shows the graph of a typical Mittag-Leffler function in this phase. The function is positive for positive $z$, and as $z \rightarrow-\infty$, the function oscillates with increasing amplitude crossing the negative $z$ axis an infinite number of times.

It has been shown (i.e., Dzhrbashyan [20] and Djrbashian [21]) that all zeros of the function $E_{2, \beta}(z)$ for $1<\beta<3$ are negative, real, and simple. This was later extended by Ostrovskii and Peresyolkova [22] to include $0<\beta<3$.
TABLE 3: Reference values in phase $[f / \infty]$.

(a) Reference value transition points along the line $\alpha=\beta$

\begin{tabular}{|c|c|}
\hline Transition & Alpha \\
\hline 1 to 3 zeros & 1.3395747103 \\
\hline 3 to 5 & 1.4892313259 \\
\hline 5 to 7 & 1.5725808412 \\
\hline 7 to 9 & 1.6280321346 \\
\hline 9 to 11 & 1.6684183137 \\
\hline 11 to 13 & 1.6995219670 \\
\hline 13 to 15 & 1.7244096473 \\
\hline 15 to 17 & 1.7448875732 \\
\hline 17 to 19 & 1.7621012104 \\
\hline 19 to 21 & 1.7768180976 \\
\hline 21 to 23 & 1.7895749696 \\
\hline 23 to 25 & 1.8007601937 \\
\hline 25 to 27 & 1.8106627476 \\
\hline 27 to 29 & 1.8195027301 \\
\hline 29 to 31 & 1.8274511290 \\
\hline 31 to 33 & 1.8346430576 \\
\hline 33 to 35 & 1.8411868715 \\
\hline 35 to 37 zeros & 1.8471706026 \\
\hline 37 to 39 & 1.8526665973 \\
\hline 39 to 41 & 1.8577349238 \\
\hline 41 to 43 & 1.8624259162 \\
\hline 43 to 45 & 1.8667821039 \\
\hline 45 to 47 & 1.8708396938 \\
\hline 47 to 49 & 1.8746297224 \\
\hline 49 to 51 & 1.8781789624 \\
\hline 51 to 53 & 1.8815106427 \\
\hline 53 to 55 & 1.8846450251 \\
\hline 55 to 57 & 1.8875998709 \\
\hline 57 to 59 & 1.8903908216 \\
\hline 59 to 61 & 1.8930317103 \\
\hline 61 to 63 & 1.8955348200 \\
\hline 63 to 65 & 1.8979110982 \\
\hline 65 to 67 & 1.9001703362 \\
\hline 67 to 69 & 1.9023213199 \\
\hline 69 to 71 zeros & 1.9043719579 \\
\hline 71 to 73 & 1.9063293896 \\
\hline 73 to 75 & 1.9082000780 \\
\hline 75 to 77 & 1.9099898892 \\
\hline 77 to 79 & 1.9117041604 \\
\hline 79 to 81 & 1.9133477593 \\
\hline 81 to 83 & 1.9149251354 \\
\hline 83 to 85 & 1.9164403642 \\
\hline 85 to 87 & 1.9178971868 \\
\hline 87 to 89 & 1.9192990435 \\
\hline 89 to 91 & 1.9206491039 \\
\hline 91 to 93 & 1.9219502937 \\
\hline 93 to 95 & 1.9232053177 \\
\hline 95 to 97 & 1.9244166806 \\
\hline
\end{tabular}


(a) Continued.

\begin{tabular}{lc}
\hline Transition & Alpha \\
\hline 97 to 99 & 1.9255867056 \\
99 to 101 & 1.9267175505 \\
101 to 103 & 1.9278112225 \\
\hline
\end{tabular}

(b) Reference value transition points along the horizontal line $\beta=1$

\begin{tabular}{|c|c|}
\hline Transition & Alpha \\
\hline 1 to 3 zeros & 1.4221906908 \\
\hline 3 to 5 & 1.5718839229 \\
\hline 5 to 7 & 1.6490682373 \\
\hline 7 to 9 & 1.6985162237 \\
\hline 9 to 11 & 1.7336930327 \\
\hline 11 to 13 & 1.7603388117 \\
\hline 13 to 15 & 1.7813926516 \\
\hline 15 to 17 & 1.7985433447 \\
\hline 17 to 19 & 1.8128419490 \\
\hline 19 to 21 & 1.8249822706 \\
\hline 21 to 23 & 1.8354435176 \\
\hline 23 to 25 & 1.8445688178 \\
\hline 25 to 27 & 1.8526111866 \\
\hline 27 to 29 & 1.8597618108 \\
\hline 29 to 31 & 1.8661681768 \\
\hline 31 to 33 & 1.8719460965 \\
\hline 33 to 35 & 1.8771879211 \\
\hline 35 to 37 zeros & 1.8819682945 \\
\hline 37 to 39 & 1.8863482727 \\
\hline 39 to 41 & 1.8903783311 \\
\hline 41 to 43 & 1.8941005978 \\
\hline 43 to 45 & 1.8975505379 \\
\hline 45 to 47 & 1.9007582408 \\
\hline 47 to 49 & 1.9037494173 \\
\hline 49 to 51 & 1.9065461804 \\
\hline 51 to 53 & 1.9091676623 \\
\hline 53 to 55 & 1.9116305080 \\
\hline 55 to 57 & 1.9139492725 \\
\hline 57 to 59 & 1.9161367439 \\
\hline 59 to 61 & 1.9182042070 \\
\hline 61 to 63 & 1.9201616614 \\
\hline 63 to 65 & 1.9220180019 \\
\hline 65 to 67 & 1.9237811690 \\
\hline 67 to 69 & 1.9254582752 \\
\hline 69 to 71 zeros & 1.9270557120 \\
\hline 71 to 73 & 1.9285792400 \\
\hline 73 to 75 & 1.9300340659 \\
\hline 75 to 77 & 1.9314249085 \\
\hline 77 to 79 & 1.9327560555 \\
\hline 79 to 81 & 1.9340314117 \\
\hline 81 to 83 & 1.9352545420 \\
\hline 83 to 85 & 1.9364287076 \\
\hline 85 to 87 & 1.9375568985 \\
\hline 87 to 89 & 1.9386418610 \\
\hline 89 to 91 & 1.9396861230 \\
\hline
\end{tabular}

(b) Continued.

\begin{tabular}{lc}
\hline Transition & Alpha \\
\hline 91 to 93 & 1.9406920148 \\
93 to 95 & 1.9416616889 \\
95 to 97 & 1.9425971365 \\
97 to 99 & 1.9435002028 \\
99 to 101 & 1.9443725998 \\
101 to 103 & 1.9452159190 \\
\hline
\end{tabular}

(c) Reference value transition points along the line $\beta=\alpha-1$

\begin{tabular}{|c|c|}
\hline Transition & Alpha \\
\hline 2 to 4 zeros & 1.2916629732 \\
\hline 4 to 6 & 1.4337910571 \\
\hline 6 to 8 & 1.5175792520 \\
\hline 8 to 10 & 1.5751778645 \\
\hline 10 to 12 & 1.6180496252 \\
\hline 12 to 14 & 1.6515923598 \\
\hline 14 to 16 & 1.6787594800 \\
\hline 16 to 18 & 1.7013316816 \\
\hline 18 to 20 & 1.7204590356 \\
\hline 20 to 22 & 1.7369236242 \\
\hline 22 to 24 & 1.7512791072 \\
\hline 24 to 26 & 1.7639303013 \\
\hline 26 to 28 & 1.7751811559 \\
\hline 28 to 30 & 1.7852650145 \\
\hline 30 to 32 & 1.7943644328 \\
\hline 32 to 34 & 1.8026245763 \\
\hline 34 to 36 & 1.8101625260 \\
\hline 36 to 38 zeros & 1.8170738953 \\
\hline 38 to 40 & 1.8234376334 \\
\hline 40 to 42 & 1.8293195759 \\
\hline 42 to 44 & 1.8347751111 \\
\hline 44 to 46 & 1.8398512130 \\
\hline 46 to 48 & 1.8445880105 \\
\hline 48 to 50 & 1.8490200131 \\
\hline 50 to 52 & 1.8531770789 \\
\hline 52 to 54 & 1.8570851871 \\
\hline 54 to 56 & 1.8607670588 \\
\hline 56 to 58 & 1.8642426610 \\
\hline 58 to 60 & 1.8675296189 \\
\hline 60 to 62 & 1.8706435553 \\
\hline 62 to 64 & 1.8735983713 \\
\hline 64 to 66 & 1.8764064818 \\
\hline 66 to 68 & 1.8790790112 \\
\hline 68 to 70 & 1.8816259606 \\
\hline 70 to 72 zeros & 1.8840563472 \\
\hline 72 to 74 & 1.8863783254 \\
\hline 77 to 76 & 1.8885992882 \\
\hline 76 to 78 & 1.8907259561 \\
\hline 78 to 80 & 1.8927644526 \\
\hline 80 to 82 & 1.8947203703 \\
\hline 82 to 84 & 1.8965988280 \\
\hline 84 to 86 & 1.8984045205 \\
\hline
\end{tabular}


(c) Continued.

\begin{tabular}{lc}
\hline Transition & Alpha \\
\hline 86 to 88 & 1.9001417625 \\
88 to 90 & 1.9018145263 \\
90 to 92 & 1.9034264764 \\
92 to 94 & 1.9049809984 \\
94 to 96 & 1.9064812261 \\
96 to 98 & 1.9079300641 \\
98 to 100 & 1.9093302094 \\
100 to 102 & 1.9106841692 \\
102 to 104 & 1.9119942782 \\
\hline
\end{tabular}

(d) Reference value transition points along the horizontal line $\beta=0$

\begin{tabular}{|c|c|}
\hline Transition & Alpha \\
\hline 2 to 4 zeros & 1.3395747103 \\
\hline 4 to 6 & 1.4892313259 \\
\hline 6 to 8 & 1.5725808412 \\
\hline 8 to 10 & 1.6280321346 \\
\hline 10 to 12 & 1.6684183137 \\
\hline 12 to 14 & 1.6995219670 \\
\hline 14 to 16 & 1.7244096473 \\
\hline 16 to 18 & 1.7448875732 \\
\hline 18 to 20 & 1.7621012104 \\
\hline 20 to 22 & 1.7768180976 \\
\hline 22 to 24 & 1.7895749696 \\
\hline 24 to 26 & 1.8007601937 \\
\hline 26 to 28 & 1.8106627476 \\
\hline 28 to 30 & 1.8195027301 \\
\hline 30 to 32 & 1.8274511290 \\
\hline 32 to 34 & 1.8346430576 \\
\hline 34 to 36 & 1.8411868715 \\
\hline 36 to 38 zeros & 1.8471706026 \\
\hline 38 to 40 & 1.8526665973 \\
\hline 40 to 42 & 1.8577349238 \\
\hline 42 to 44 & 1.8624259162 \\
\hline 44 to 46 & 1.8667821039 \\
\hline 46 to 48 & 1.8708396938 \\
\hline 48 to 50 & 1.8746297224 \\
\hline 50 to 52 & 1.8781789624 \\
\hline 52 to 54 & 1.8815106427 \\
\hline 54 to 56 & 1.8846450251 \\
\hline 56 to 58 & 1.8875998709 \\
\hline 58 to 60 & 1.8903908216 \\
\hline 60 to 62 & 1.8930317103 \\
\hline 62 to 64 & 1.8955348200 \\
\hline 64 to 66 & 1.8979110982 \\
\hline 66 to 68 & 1.9001703362 \\
\hline 68 to 70 & 1.9023213199 \\
\hline 70 to 72 zeros & 1.9043719579 \\
\hline 72 to 74 & 1.9063293896 \\
\hline 77 to 76 & 1.9082000780 \\
\hline 76 to 78 & 1.9099898892 \\
\hline 78 to 80 & 1.9117041604 \\
\hline
\end{tabular}

(d) Continued.

\begin{tabular}{lc}
\hline Transition & Alpha \\
\hline 80 to 82 & 1.9133477593 \\
82 to 84 & 1.9149251354 \\
84 to 86 & 1.9164403642 \\
86 to 88 & 1.9178971868 \\
88 to 90 & 1.9192990435 \\
90 to 92 & 1.9206491039 \\
92 to 94 & 1.9219502937 \\
94 to 96 & 1.9232053177 \\
96 to 98 & 1.9244166806 \\
98 to 100 & 1.9255867056 \\
100 to 102 & 1.9267175505 \\
102 to 104 & 1.9278112225 \\
\hline
\end{tabular}

(e) Reference value transition points along the horizontal line $\beta=2$

\begin{tabular}{|c|c|}
\hline Transition & Alpha \\
\hline 2 to 4 zeros & 1.7100323859 \\
\hline 4 to 6 & 1.7651251779 \\
\hline 6 to 8 & 1.7997959826 \\
\hline 8 to 10 & 1.8241639279 \\
\hline 10 to 12 & 1.8424530845 \\
\hline 12 to 14 & 1.8567978278 \\
\hline 14 to 16 & 1.8684122443 \\
\hline 16 to 18 & 1.8780456420 \\
\hline 18 to 20 & 1.8861890746 \\
\hline 20 to 22 & 1.8931795541 \\
\hline 22 to 24 & 1.8992570236 \\
\hline 24 to 26 & 1.9045975105 \\
\hline 26 to 28 & 1.9093334138 \\
\hline 28 to 30 & 1.9135664414 \\
\hline 30 to 32 & 1.9173761529 \\
\hline 32 to 34 & 1.9208257688 \\
\hline 34 to 36 & 1.9239662228 \\
\hline 36 to 38 zeros & 1.9268390530 \\
\hline 38 to 40 & 1.9294785049 \\
\hline 40 to 42 & 1.9319130876 \\
\hline 42 to 44 & 1.9341667441 \\
\hline 44 to 46 & 1.9362597431 \\
\hline 46 to 48 & 1.9382093682 \\
\hline 48 to 50 & 1.9400304552 \\
\hline 50 to 52 & 1.9417358171 \\
\hline 52 to 54 & 1.9433365818 \\
\hline 54 to 56 & 1.9448424643 \\
\hline 56 to 58 & 1.9462619872 \\
\hline 58 to 60 & 1.9476026606 \\
\hline 60 to 62 & 1.9488711300 \\
\hline 62 to 64 & 1.9500732990 \\
\hline 64 to 66 & 1.9512144314 \\
\hline 66 to 68 & 1.9522992368 \\
\hline 68 to 70 & 1.9533319424 \\
\hline 70 to 72 zeros & 1.9543163543 \\
\hline 72 to 74 & 1.9552559090 \\
\hline
\end{tabular}


(e) Continued.

\begin{tabular}{lc}
\hline Transition & Alpha \\
\hline 77 to 76 & 1.9561537182 \\
76 to 78 & 1.9570126063 \\
78 to 80 & 1.9578351437 \\
80 to 82 & 1.9586236746 \\
82 to 84 & 1.9593803423 \\
84 to 86 & 1.9601071098 \\
86 to 88 & 1.9608057791 \\
88 to 90 & 1.9614780074 \\
90 to 92 & 1.9621253217 \\
92 to 94 & 1.9627491312 \\
94 to 96 & 1.9633507390 \\
96 to 98 & 1.9639313515 \\
98 to 100 & 1.9644920877 \\
100 to 102 & 1.9650339870 \\
102 to 104 & 1.9655580163 \\
\hline
\end{tabular}

The point $\alpha=2, \beta=3$ was not included because the zeros of $E_{2,3}(z)$ are not simple but have a multiplicity of 2 . Nevertheless it is still true that the zeros of $E_{2,3}(z)$ are negative and real. For $\beta>3$, it has been shown (Popov and Sedletski $[10])$ that $E_{2, \beta}(z)$ has no real zeros and thus belongs to phase $[0, \infty]$. Thus Mittag-Leffler functions on the line $\alpha=2$ for $0<\beta \leq 3$ belong to the phase $[0 / \infty]$ as indicated by the arrow in Figure 1.

There is considerable theoretical work searching for all pairs $(\alpha, \beta)$ such that the zeros of the Mittag-Leffler functions are negative, real, and simple (i.e., Popov [23-26], Popov and Sedletski [10], and Ostrovskii and Peresyolkova [22]). For example, Popov [25] showed that for $\alpha>2$ and $0<\beta \leq(2 \alpha-$ 1) all zeros of $E_{\alpha, \beta}(z)$ are negative, real, and simple. Although this covers the majority of phase $[\infty / 0]$ the upper limit on $\beta$ is more restrictive than (8) predicts. However, the work of Popov and others is also more restrictive requiring the zeros to be simple. The phases proposed in Figure 1 are only concerned with whether the zeros are real or complex. The general properties attributed to the Mittag-Leffler functions in each phase hold true regardless of whether the zeros are simple or multiples.

Although the phase diagram in Figure 1 is restricted to

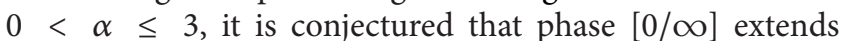
to $2 \leq \alpha \leq \infty$ based on the theoretical work of Popov and the others listed above. Also supporting this hypothesis is the work of Wiman [16], Pólya [27], and Ostrovskii and Peresyokkova [22] who have shown that all zeros of $E_{\alpha, 1}(z)$ for $\alpha \geq 2$ are real, negative, and simple. In addition, Popov [26] has shown that all zeros of the function $E_{N, N+1}(z)$ are real, negative, and simple for $N \geq 3$ and that all zeros of $E_{4,9}(z)$ are real, negative, and simple.

\section{Phase $[\infty / f]$}

This phase extends from $2<\alpha \leq 3$ and for $\beta$ above the phase boundary line given approximately by (8). Figure 13 shows a typical Mittag-Leffler function in this phase.

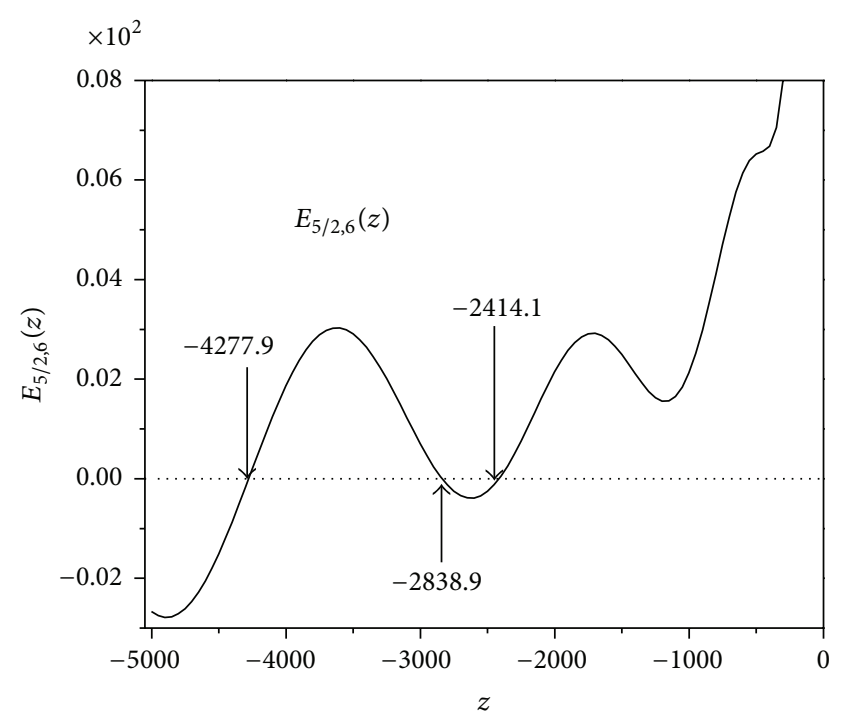

FIgure 13: Typical Mittag-Leffler function in $[\infty / f]$.

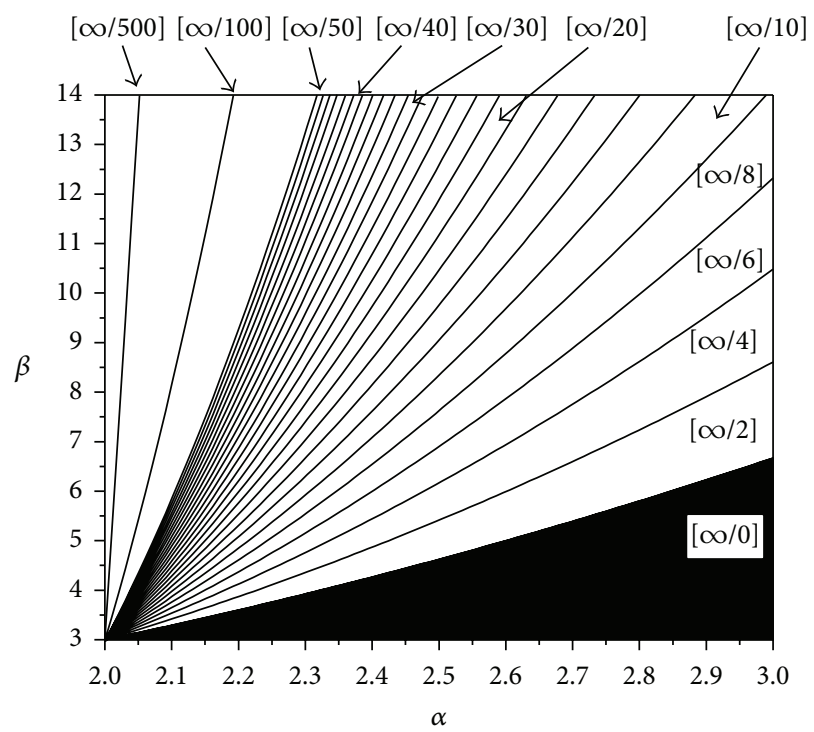

FIGURE 14: Phase $[\infty / f]$ in detail showing subphases $[\infty / n], n=$ $2,4,6, \ldots$

The function is positive for positive $z$ and as $z \rightarrow-\infty$ the function oscillates with growing amplitude crossing the negative real axis an infinite number of times. The MittagLeffler functions in phase $[\infty / f]$ differ from those in phase $[\infty / 0]$ in that the functions in phase $[\infty / f]$ exhibit a finite number of oscillations whose relative minima are above the negative $z$-axis, whereas for functions in phase $[\infty / 0]$, the relative minima for all oscillations are below the negative $z$-axis (see Figure 12). For fixed $\alpha$, and $\beta$ incrementally above the phase boundary line, the relative minimum in the oscillation nearest $z=0$ no longer lies below the negative $z$ axis but is now above the negative $z$-axis and thus the function has lost two real zeros and gained two complex zeros (one and its complex conjugate). The phase boundary occurs at 
TABLE 4: Reference values in phase $[\infty / f]$.

(a) Reference points on the phase boundary line separating phases [ $[\infty / 2]$ from $[\infty / 4]$

\begin{tabular}{lc}
\hline$\alpha$ & $\beta$ \\
\hline 2 & 3 \\
2.2276114869 & 4 \\
2.4245980282 & 5 \\
2.6013352823 & 6 \\
2.7631879843 & 7 \\
2.9134444409 & 8 \\
3 & 8.6072783765 \\
\hline
\end{tabular}

(b) Reference points on the phase boundary line separating phases [ $\infty / 4]$ from $[\infty / 6]$

\begin{tabular}{lc}
\hline$\alpha$ & $\beta$ \\
\hline 2 & 3 \\
2.1799836579 & 4 \\
2.3364037665 & 5 \\
2.4775449443 & 6 \\
2.6074522928 & 7 \\
2.7285611696 & 8 \\
2.8425033406 & 9 \\
2.9504465415 & 10 \\
3 & 10.4761755002 \\
\hline
\end{tabular}

(c) Reference points on the phase boundary line separating phases [o/6] from $[\infty / 8]$

\begin{tabular}{lc}
\hline$\alpha$ & $\beta$ \\
\hline 2 & 3 \\
2.1504894698 & 4 \\
2.2816699180 & 5 \\
2.4005387002 & 6 \\
2.5103738032 & 7 \\
2.6131154583 & 8 \\
2.7100556595 & 9 \\
2.8021181175 & 10 \\
2.8899956024 & 11 \\
2.9742260037 & 12 \\
3 & 12.3140908740 \\
\hline
\end{tabular}

(d) Reference Points on the phase boundary line separating phases [o/8] from $[\infty / 10]$

\begin{tabular}{lc}
\hline$\alpha$ & $\beta$ \\
\hline 2 & 3 \\
2.1301385540 & 4 \\
2.2438414409 & 5 \\
2.3472084518 & 6 \\
2.4430135894 & 7 \\
2.5328752160 & 8 \\
2.6178636961 & 9 \\
2.6987423420 & 10 \\
2.7760835480 & 11 \\
2.8503322845 & 12 \\
\hline
\end{tabular}

(d) Continued.

\begin{tabular}{lc}
\hline$\alpha$ & $\beta$ \\
\hline 2.9218440026 & 13 \\
2.9909087735 & 14 \\
3 & 14.1341324772 \\
\hline
\end{tabular}

the value of $\beta$ when this relative minimum rests on the negative $z$-axis. As $\beta$ is increased further, the relative minimum of the second oscillation nearest $z=0$ also occurs above the negative $z$-axis (shown in Figure 13) admitting two more complex zeros for a total of 4 complex zeros and an infinite number of real zeros. This process continues as $\beta$ is increased; each time the relative minimum of an oscillation moves above the negative $z$-axis the function loses two real zeros and gains two complex zeros. This phase has been extensively mapped by the authors and is shown in Figure 14. Reference values on the subphase boundary lines are given in Table 4 .

Note that all Mittag-Leffler functions in this phase just above the boundary line have 2 complex zeros and thus this region is listed as $[\infty / 2]$. Since each of these regions $[\infty / 2],[\infty / 4],[\infty / 6], \ldots$ all has the same general behavior, they are considered as one major phase $[\infty / f]$ with an infinite number of subphases. An interesting observation from Figure 14 is that the phase $[\infty / \infty]$ can never be achieved but is only approached for $\beta>3$ and $\alpha=2+\varepsilon$ as $\varepsilon$ becomes incrementally small.

\section{Summary}

The depiction of the $\alpha$ - $\beta$ phase diagram for the Mittag-Leffler functions with real arguments is represented by Figure 1 with subphases shown in more detail in Figures 7, 8, 9, 10, and 14. Each phase represents a region where $E_{\alpha, \beta}(z)$ have not only the same type of zeros but also exhibit similar functional behavior. This represents a major step forward in characterizing the nature of the zeros and organizing the Mittag-Leffler functions according to their behavior. For complex arguments, the reader is referred to the numerical calculations for $E_{\alpha, \beta}(z)$ in the complex plane by Hilfer and Seybold [28]. Their results are consistent with the present work where the efforts overlap.

\section{References}

[1] I. Podlubny, Fractional Differential Equations, vol. 198 of Mathematics in Science and Engineering, Academic Press, San Diego, Calif, USA, 1999.

[2] R. L. Magin, Fractional Calculus in Bioengineering, Begell House Publishers, Redding, Conn, USA, 2006.

[3] A. A. Kilbas, H. M. Srivastava, and J. J. Trujillo, Theory and Applications of Fractional Differential Equations, vol. 204 of North-Holland Mathematics Studies, Elsevier Science B.V., Amsterdam, The Netherlands, 2006.

[4] F. Mainardi, Fractional Calculus and Waves in Linear Viscoelasticity, Imperial College Press, London, UK, 2010.

[5] L. V. Ahlfors, Complex Analysis, McGraw-Hill, New York, NY, USA, 2nd edition, 1966. 
[6] R. Gorenflo and F. Mainardi, "Fractional calculus: integral and differential equations of fractional order," in Fractals and Fractional Calculus in Continuum Mechanics, A. Carpenteri and F. Mainardi, Eds., vol. 378 of CISM Courses and Lectures, pp. 223-276, Springer, Vienna, Austria, 1997.

[7] A. M. Sedletski, "Asymptotic formulas for zeros of functions of Mittag-Leffler type," Analysis Mathematica, vol. 20, no. 2, pp. 117-132, 1994.

[8] A. M. Sedletski, "Nonasymptotic properties of the roots of a function of Mittag-Leffler type," Matematicheskie Zametki, vol. 75, no. 3, pp. 405-420, 2004.

[9] R. Gorenflo, Yu. Luchko, and S. V. Rogozin, "Mittag-Leffler type functions: notes on growth properties and distribution of zeros," A97-04, Fachbereich Mathematik und Informatik, Freie Universitat, Berlin, Germany, 1997.

[10] A. Yu. Popov and A. M. Sedletski, "Distribution of the zeros of the Mittag-Leffler function," Doklady Mathematics, vol. 67, pp. 336-339, 2003.

[11] W. R. Schneider, "Completely monotone generalized MittagLeffler functions," Expositiones Mathematicae, vol. 14, no. 1, pp. 3-16, 1996.

[12] K. S. Miller and S. G. Samko, "A note on the complete monotonicity of the generalized Mittag-Leffler function," Real Analysis Exchange, vol. 23, no. 2, pp. 753-755, 1997/98.

[13] K. S. Miller and S. G. Samko, "Completely monotonic functions," Integral Transforms and Special Functions, vol. 12, no. 4, pp. 389-402, 2001.

[14] T. S. Aleroev and H. T. Aleroeva, "A problem on the zeros of the Mittag-Leffler function and the spectrum of a fractional-order differential operator," Electronic Journal of Qualitative Theory of Differential Equations, vol. 25, pp. 1-18, 2009.

[15] R. Gorenflo and F. Mainardi, "Fractional oscillations and Mittag-Leffler functions," in Proceedings of the International Workshop on the Recent Advances in Applied Mathematics (RAAM '96), Kuwait University, Kuwait City, Kuwait, 1996.

[16] A. Wiman, "Über die Nullstellen der Funktionen $\mathrm{E}_{\alpha}(\mathrm{x})$," Acta Mathematica, vol. 29, no. 1, pp. 217-234, 1905.

[17] J. W. Hanneken, D. M. Vaught, and B. N. N. Achar, "Enumeration of the real zeros of the Mittag-Leffler function $\mathrm{E}_{\alpha}(\mathrm{x})$, $1<\alpha<2$," in Advances in Fractional Calculus: Theoretical Developments and Applications in Physics and Engineering, J. Sabatier, O. P. Agrawal, and J. A. T. Machado, Eds., pp. 15-26, Springer, Dordrecht, The Netherlands, 2007.

[18] J. W. Hanneken, B. N. N. Achar, D. M. Vaught, S. T. Spencer, and T. R. Ensley, "An alpha-beta phase diagram representation of the zeros and properties of the Mittag-Leffler function," in Proceedings of the International Workshop on Fractional Differentiation and its Applications (FDA '10), University of Extremadura, Badajoz, Spain, October 2010.

[19] J. S. Duan, Z. Wang, Y. L. Liu, and X. Qiu, "Eigenvalue problems for fractional ordinary differential equations," Chaos, Solitons \& Fractals, vol. 46, pp. 46-53, 2013.

[20] M. M. Dzhrbashyan, "Interpolation and spectral expansions associated with differential operators of fractional order," Soviet Journal of Contemporary Mathematical Analysis, vol. 19, no. 2, pp. 1-116, 1984.

[21] M. M. Djrbashian, Harmonic Analysis and Boundary Value Problems in the Complex Domain, vol. 65 of Operator Theory: Advances and Applications, Birkhäuser, Basel, Switzerland, 1993.

[22] I. V. Ostrovskii and I. N. Peresyolkova, "Nonasymptotic results on distribution of zeros of the function $\mathrm{E}_{\rho}(\mathrm{z}, \mu)$," Analysis Mathematica, vol. 23, no. 4, pp. 283-296, 1997.
[23] A. Y. Popov, "On the Ostrovskii-Peresyolkova conjecture about zeros of the Mittag-Leffler functions," Proceedings of the Steklov Institute of Mathematics, supplement 1, pp. S167-S182, 2001.

[24] A. Y. Popov and A. M. Sedletski, "Distribution of the zeros of the Mittag-Leffler function," Doklady Mathematics, vol. 67, pp. 336-339, 2003.

[25] A. Y. Popov, "On zeros of Mittag-Leffler functions with parameter $\rho<1 / 2$," Analysis Mathematica, vol. 32, no. 3, pp. 207-246, 2006.

[26] A. Y. Popov, "On zeros of a certain family of Mittag-Leffler functions," Journal of Mathematical Sciences, vol. 144, no. 4, pp. 4228-4231, 2005.

[27] G. Pólya, "Bemerkung über die Mittag-Lefflerschen Funktionen," Tôhoku Mathematical Journal, vol. 19, pp. 241-248, 1921.

[28] R. Hilfer and H. J. Seybold, "Computation of the generalized Mittag-Leffler function and its inverse in the complex plane," Integral Transforms and Special Functions, vol. 17, no. 9, pp. 637652, 2006. 


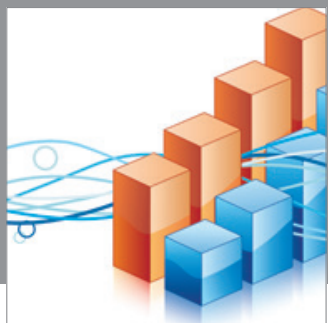

Advances in

Operations Research

mansans

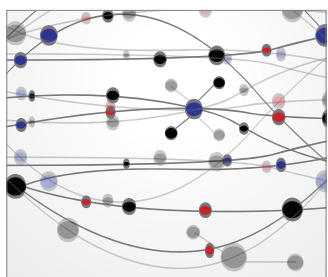

The Scientific World Journal
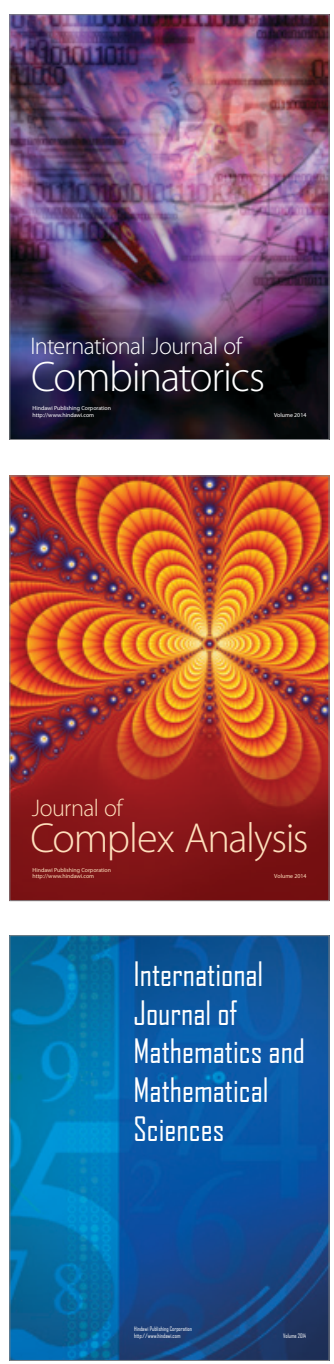
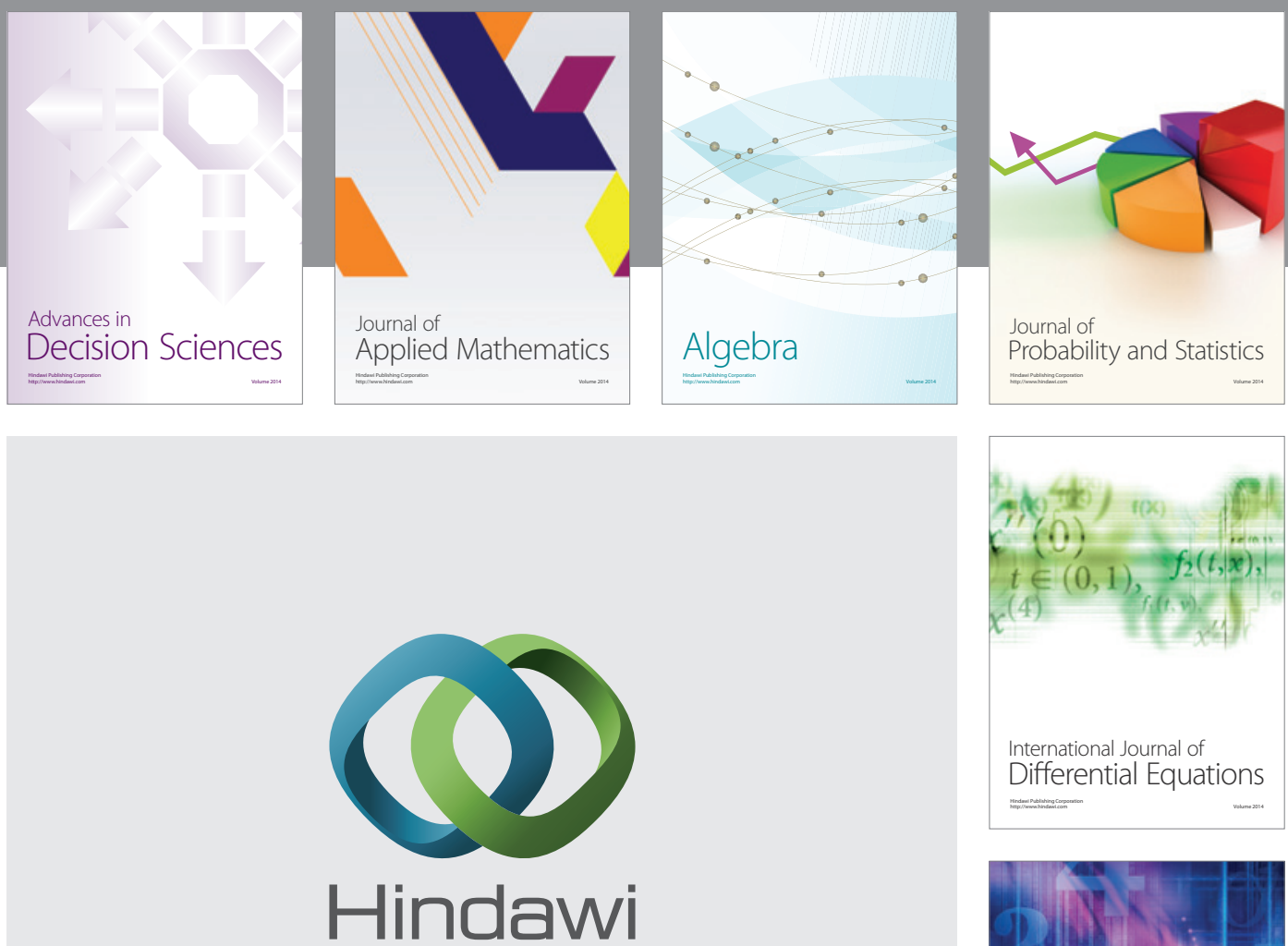

Submit your manuscripts at http://www.hindawi.com
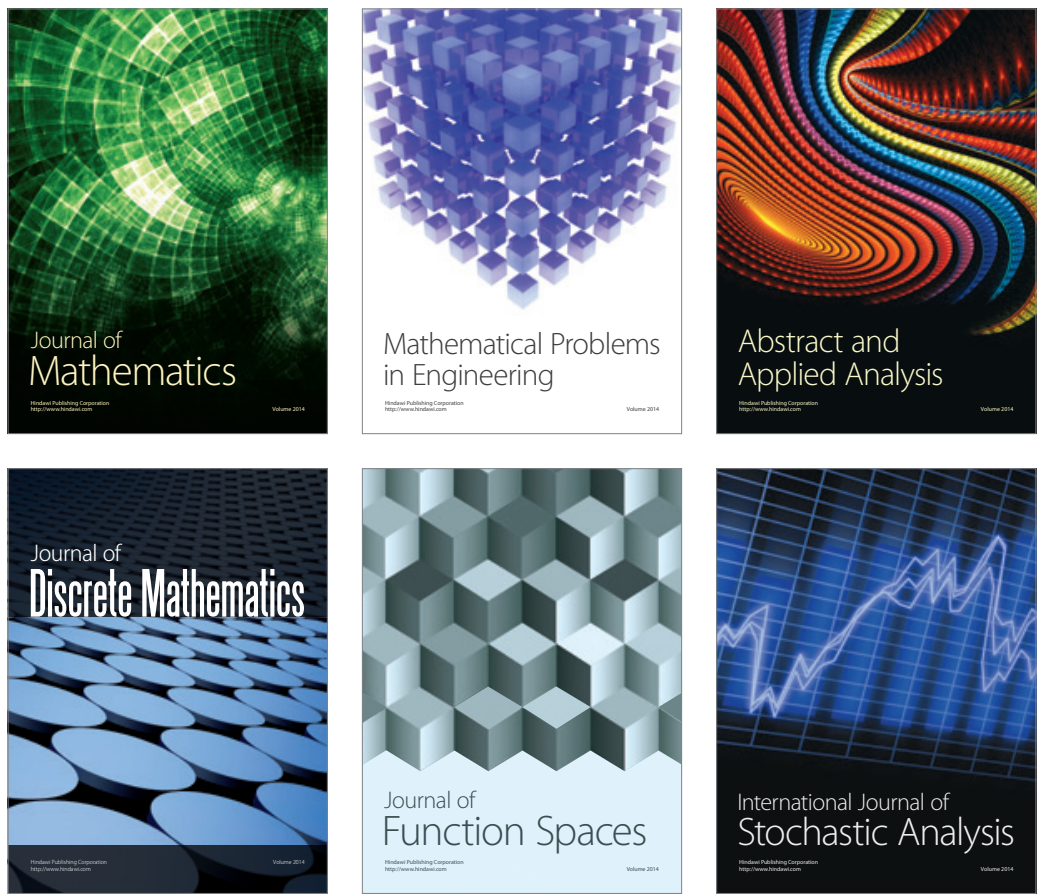

Journal of

Function Spaces

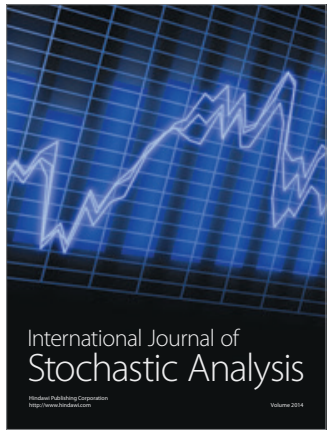

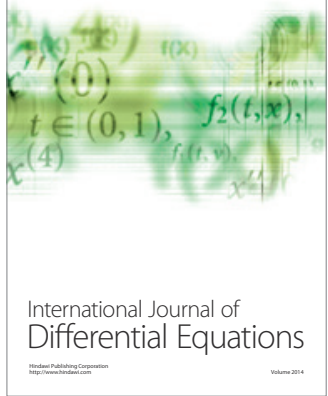
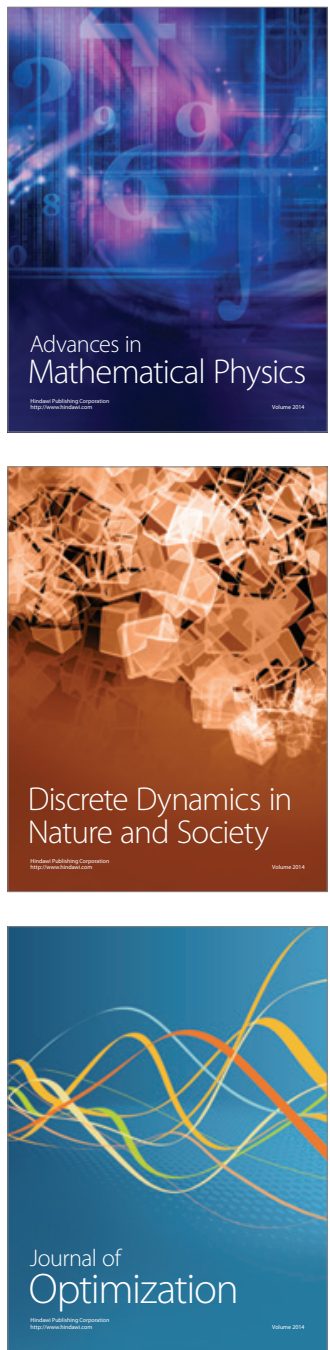